ISSN 1855-3966 (printed edn.), ISSN 1855-3974 (electronic edn.)

ARS MATHEMATICA CONTEMPORANEA 20 (2021) 195-197

https://doi.org/10.26493/1855-3974.2334.331

(Also available at http://amc-journal.eu)

\title{
A simple and elementary proof of Whitney's unique embedding theorem
}

\author{
Gunnar Brinkmann \\ Applied Mathematics, Computer Science and Statistics, Ghent University, Belgium
}

Received 13 May 2020, accepted 12 February 2021, published online 27 October 2021

\begin{abstract}
In this note we give a short and elementary proof of a more general version of Whitney's theorem that 3-connected planar graphs have a unique embedding in the plane. A consequence of the theorem is also that cubic plane graphs cannot be embedded in a higher genus with a simple dual. The aim of this paper is to promote a simple and elementary proof, which is especially well suited for lectures presenting Whitney's theorem.
\end{abstract}

Keywords: Polyhedra, graph, embedding.

Math. Subj. Class. (2020): 05C10, 57M60, 57M15

\section{Introduction}

Whitney's famous unique embedding theorem has been formulated in various equivalent forms. One form is that the facial cycles of 3-connected graphs embedded in the plane are well determined, so that for any two embeddings there is a graph isomorphism between the duals. Another is (implied by the Jordan-Schönflies Theorem) that any two topological embeddings of a graph on the sphere can be mapped onto each other by a homeomorphism of the sphere that maps the two images of a vertex onto each other.

We will formulate the theorem and describe the proof in the language of combinatorial embeddings in oriented closed surfaces. For the translation to the language of topological 2-cell embeddings, methods from standard books like [1] or [3] can be used.

We interpret each edge $\{u, v\}$ of an undirected embedded graph $G$ as two directed edges: $e=(u, v)$ and its inverse $e^{-1}=(v, u)$. An embedded graph in an oriented closed surface is a graph where for every vertex $u$ there is a cyclic order of all edges $(u,$.$) (usually$ called a rotation). The cyclic ordering defines the orientation around the vertex. We write

*I would like to thank Bojan Mohar for pointing me to the earlier uses of the crossing Jordan curves argument! E-mail address: gunnar.brinkmann@ugent.be (Gunnar Brinkmann) 
$\mathrm{nx}(e)$ for the next edge in the order around the starting point of a directed edge $e$. The inverse graph or mirror image is the graph $G^{-1}$ with all cyclic orders reversed.

A face in an embedded graph $G$ is a directed cyclic walk $e_{0}, \ldots, e_{n-1}$, so that for $0 \leq i<n$ we have that $\operatorname{nx}\left(e_{i}^{-1}\right)=e_{(i+1)}(\bmod n)$. We say that the set $\{e, \operatorname{nx}(e)\}$ forms an angle of $G$ and $G^{-1}$ if one of them has a face containing $e^{-1}, \operatorname{nx}(e)$ as a subsequence. In this case the other has a face containing $\mathrm{nx}(e)^{-1}, e$. If a face is a simple cyclic walk, we call the corresponding undirected cycle also a (simple) facial cycle. We consider an embedded graph $G$ and its mirror image $G^{-1}$ as equivalent, as the faces have the same sequences of underlying undirected edges - only in reversed order. The genus of an embedded graph can be computed by the Euler formula using the number $v$ of vertices, $e$ of (undirected) edges, and $f$ of faces as $\gamma(G)=\frac{2-(v-e+f)}{2}$. We refer to a (not necessarily embedded) graph that can be embedded with genus 0 as planar and to an embedded graph with genus 0 as plane.

With this notation and concept of equivalence Whitney's famous theorem [5] can be shortly stated as:

\section{A 3-connected planar graph has an - up to equivalence - unique embedding in the plane.}

We will prove a stronger theorem using the concept of polyhedral embedding that requires some important properties of polyhedra - that is plane 3-connected graphs - but allows higher genera. It is an easy consequence of the Jordan Curve Theorem that polyhedra are polyhedral embeddings.

Definition 1.1. A polyhedral embedding of a graph $G=(V, E)$ in an oriented closed surface is an embedding so that each facial walk is a simple cycle and the intersection of any two faces is either empty, a single vertex or a single edge.

For cubic embedded graphs this is equivalent to the dual graph being simple.

The argument of crossing Jordan curves that we will use in the proof was first published by Thomassen in [4], but also known to Robertson and later used by Mohar and Robertson in [2]. See also Theorem 5.7.1 in [3]. In fact, in [4] the argument was used to prove that 3 -connected planar graphs embedded with genus $g>0$ have facewidth at most 2 . Together with Whitney's theorem, this implies Theorem 1.2. We will give every detail of the proof in order to make it well suited for lectures presenting Whitney's theorem, but the arguments are exactly the same arguments of crossing Jordan curves that Thomassen used - only that here the planar case, that is: Whitney's theorem - is included too.

Theorem 1.2. A 3-connected planar graph has an-up to equivalence-unique polyhedral embedding.

Proof. Let $G$ be a plane embedding of a 3-connected planar graph with mirror image $G^{-1}$ and let $G^{\prime}$ be an embedding different from these two. We say that a vertex of $G^{\prime}$ has type 1 if the order is the same as in $G$, type -1 if it is the same as in $G^{-1}$ and type 2 otherwise. As $G^{\prime}$ is neither $G$ nor $G^{-1}, G^{\prime}$ has a vertex of type 2 or an edge with one vertex of type 1 and one vertex of type -1 .

Assume first that there is a vertex $v$ of type 2 . Let $e_{0}, \ldots, e_{d-1}$ be the order of edges around $v$ in $G^{\prime}$. If $\left\{e_{0}, e_{1}\right\}$ is not an angle of $G$, we take this set of edges. Otherwise assume w.l.o.g. that $e_{1}=\operatorname{nx}\left(e_{0}\right)$ in $G$ and let $j$ be minimal so that in $G$ we have $\operatorname{nx}\left(e_{j}\right) \neq$ $e_{(j+1)(\bmod d)}$. As in $G^{-1}$ we have $\operatorname{nx}\left(e_{j}\right)=e_{j-1}$, the edge $e_{(j+1)(\bmod d)}$ follows $e_{j}$ neither in $G$ nor in $G^{\prime}$, so $\left\{e_{j}, e_{(j+1)}(\bmod d)\right\}$ is not an angle in $G$. W.l.o.g. assume $j=0$. 
So the order around $v$ in $G$ is $e_{0}, e_{i_{1}}, \ldots, e_{i_{j}}, e_{1}, e_{i_{j+1}}, \ldots, e_{i_{d-2}}$ with $1 \leq j<d-2$ and assume w.l.o.g. that $e_{d-1} \in\left\{e_{i_{j+1}}, \ldots, e_{i_{d-2}}\right\}$. Let $y=\max \left\{i_{1}, \ldots, i_{j}\right\}$, so $y<d-1$ and $(y+1) \in\left\{i_{j+1}, \ldots, i_{d-2}\right\}$, which implies that $\left\{e_{y}, e_{y+1}\right\}$ is an angle of $G^{\prime}$ with $e_{y} \in$ $\left\{e_{i_{1}}, \ldots, e_{i_{j}}\right\}$ and $e_{y+1} \in\left\{e_{i_{j+1}}, \ldots, e_{i_{d-2}}\right\}$. Let $F$ be the facial cycle in $G^{\prime}$ containing the angle $\left\{e_{0}, e_{1}\right\}$ and $F^{\prime}$ be the facial cycle containing $\left\{e_{y}, e_{y+1}\right\}$. We have $F \neq F^{\prime}$ as otherwise the faces would not be simple cycles. In $G$ these cycles are not facial cycles, but two Jordan curves crossing each other in $v$. Due to the Jordan curve theorem, there must be a second crossing, so $F, F^{\prime}$ are two facial cycles that have at least two vertices in common that are not endpoints of a common edge - a contradiction to $G^{\prime}$ being polyhedral.

Assume now that all vertices are of type 1 or type -1 . Then there is an edge $e_{0}$ with one vertex of type 1 and one of type -1 . Assume that in $G$ the orientation around the type 1 vertex of $e_{0}$ is $e_{0}, e_{1}, \ldots, e_{d}$ and around the type -1 vertex it is $e_{0}^{-1}, e_{1}^{\prime}, \ldots, e_{d^{\prime}}^{\prime}$, so in $G^{\prime}$ it is $e_{0}, e_{1}, \ldots, e_{d}$ resp. $e_{d^{\prime}}^{\prime}, e_{d^{\prime}-1}^{\prime}, \ldots, e_{0}^{-1}$. In $G^{\prime}$ there is a face $F$ containing $e_{d}^{-1}, e_{0}, e_{d^{\prime}}^{\prime}$ and another face $F^{\prime}$ containing $e_{1}^{\prime-1}, e_{0}^{-1}, e_{1}$. In $G$ the corresponding cycles are again no facial cycles but Jordan curves crossing each other (with one common edge), so like in the first case we get a contradiction from the fact that there must be a second intersection between $F$ and $F^{\prime}$.

As plane embeddings of 3-connected graphs are all polyhedral, this also implies Whitney's theorem, but there are also other consequences that are worth mentioning. They follow already from Theorem 8.1 in [4]. Note that for graphs with 1- or 2-cut there are no polyhedral embeddings in any closed orientable surface.

\section{Corollary 1.3.}

- There are no polyhedral embeddings of planar graphs in any orientable surface but the plane.

- There are no embeddings of cubic planar graphs with a simple dual in any orientable surface but the plane.

\section{ORCID iD}

Gunnar Brinkmann (D) https://orcid.org/0000-0003-4168-0877

\section{References}

[1] J. L. Gross and T. W. Tucker, Topological Graph Theory, Wiley-Interscience Series in Discrete Mathematics and Optimization, John Wiley \& Sons, New York, 1987.

[2] B. Mohar and N. Robertson, Planar graphs on nonplanar surfaces, J. Comb. Theory Ser. B 68 (1996), 87-111, doi:10.1006/jctb.1996.0058.

[3] B. Mohar and C. Thomassen, Graphs on Surfaces, Johns Hopkins Studies in the Mathematical Sciences, Johns Hopkins University Press, Baltimore, Maryland, 2001.

[4] C. Thomassen, Embeddings of graphs with no short noncontractible cycles, J. Comb. Theory Ser. B 48 (1990), 155-177, doi:10.1016/0095-8956(90)90115-g.

[5] H. Whitney, 2-isomorphic graphs, Amer. J. Math. 55 (1933), 245-254, doi:10.2307/2371127. 